\title{
Bacterial Profile And Antibiotic Susceptibility Pattern Of Urinary Tract Infection Among Children Attending Felege Hiwot Referral Hospital, Bahir Dar, Northwest Ethiopia
}

This article was published in the following Dove Press journal:

Infection and Drug Resistance

Yerega Belete'

Daniel Asrat ${ }^{2}$

Yimtubezinash

Woldeamanuel (D) ${ }^{2}$

Gebeyehu Yihenew'

Addisu Gize'

'Department of Microbiology, St. Paul's Hospital Millennium Medical College,

Addis Ababa, Ethiopia; ${ }^{2}$ Department of

Microbiology, Immunology And

Parasitology, Faculty of Medicine, Addis

Ababa University, Addis Ababa, Ethiopia
Correspondence: Yerega Belete St. Paul's Hospital Millennium Medical College, Addis Ababa, Ethiopa Tel +251920735677

Email yereb2000@gmail.com
Background: Urinary tract infection (UTI) is a common and important clinical problem in pediatrics. Recurrent UTIs may lead to renal scarring, hypertension, and end-stage renal dysfunction later in life. The objective of the study was to determine bacterial profile and antimicrobial susceptibility pattern of urinary tract infections (UTIs) among children attending Felege Hiwot Referral Hospital (FHRH).

Methods: A cross-sectional study was conducted from February 2013 to May 2013 among children 5-15 years of age with symptoms of UTI. Samples were processed for culture and identification. Antimicrobial susceptibility was done for positive urine cultures by the KirbyBauer's disk diffusion method based on standards of the Clinical Laboratory Standard Institute (CLSI). Data were entered into Epi-data version 3.2.1 and exported to the Statistical Package for the Social Science (SPSS) version 20 statistical software. Fisher's exact test and binary logistic regression test results were used.

Results: A total of 259 urine samples were collected from children with UTI. The result revealed $41(15.8 \%)$ samples had significant bacteriuria, among which the most prevalent pathogen was E. coli 14 (34.1\%) followed by Pseudomonas species. Gram-negative bacteria showed high level of sensitivity to ciprofloxacin (70), norfloxacin $(63.4 \%)$ and ceftriaxone $(60 \%)$, whereas the level of resistance was high to ampicillin $(80 \%)$ and nitrofurantoin $(70 \%)$. Gram-positive isolates showed high sensitivity to ciprofloxacin $(77.8 \%)$, penicillin $(72.8 \%)$ and erythromycin (72.7\%). Multiple drug resistance (MDR) for Gram-positive and Gram-negative bacteria was $100 \%$ and $83.1 \%$, respectively.

Conclusion: $E$. coli is the predominant bacteria isolated in the present study. The results showed that the prevalence of resistance to at least one antibiotic to commonly prescribed antimicrobials was high. Hence, the guidelines for empiric treatment of UTI should be reevaluated periodically based on local studies.

Keywords: urinary tract infection, significant bacteriuria, antimicrobial susceptibility, children

\section{Background}

Urinary tract infection (UTI) is a term applied to a variety of clinical conditions ranging from asymptomatic presence. Worldwide, about 150 million people are diagnosed with UTI each year, costing the global economy in excess of 6 billion US dollars. ${ }^{1}$ It has also become the most common hospital-acquired infection, accounting for as many as $35 \%$ of nosocomial infections, and they are the second most common cause of bacteraemia in hospitalized patients. $^{2}$ 
Urinary tract infection is a common and important clinical problem in pediatrics, as recurrent UTIs may lead to renal scarring, hypertension and end-stage renal dysfunction later in life. UTIs account for $0.7 \%$ of physician office visits and 5-14\% of emergency departments visits by children annually. Diagnosis in younger children may be difficult, however, older children often complain of urinary symptoms that are classically associated with UTI, such as dysuria, frequency, urgency and may present with suprapubic tenderness on examination. ${ }^{3}$ Since childhood UTI is the most important factor affecting adult health, children should be treated immediately with appropriate antibiotics. ${ }^{4}$

Antimicrobial therapy is often initiated empirically before the urine culture results are available. Furthermore, UTIs are often treated with broad-spectrum antibiotics when one with a narrow spectrum of activity may be appropriate because of concerns about infection with resistant organisms. The etiology of UTI and the antibiotic resistance of uropathogens have been changing over the past years, both in community and nosocomial infection. ${ }^{5,6}$

Given there are both geographical and historical variability of antimicrobial resistance patterns, the knowledge of the sensitivity pattern of common uropathogens according to local epidemiological studies is necessary for selection of an appropriate antibiotic for empirical treatment. Furthermore, studies recommended that the policies for treatment of UTI in children should be re-evaluated every 5 years according to local resistance rates. ${ }^{7}$

There are studies on bacterial profile and antibiotic susceptibility pattern of UTI in different parts of Ethiopia, but these have been conducted mostly on adults and pregnant women. ${ }^{8,9}$ To our knowledge, there has been no study done around our targeted age group (age 5-15) focused on understanding the antimicrobial resistance pattern of pathogens are more frequently isolated in UTI locally. Consequently, studies on the assessment of bacterial profile and antibiotic susceptibility pattern of UTI in children in our geographical area (Bahir Dar) were limited. Hence, this study aimed to determine the bacterial profile and antibiotic susceptibility pattern of urinary tract infection among children attending at Felege Hiwot Referral Hospital, Bahir Dar, Northwest Ethiopia.

\section{Methods And Materials}

\section{Study Setting And Period}

The study was conducted from February 2013 to May 2013 at Felege Hiwot Referral Hospital, Bahir Dar,
Amhara, Northwest Ethiopia. Bahir Dar is the capital city of Amhara region with total population of 155,428 according to 2007 census. The hospital includes inpatient, outpatient, and emergency wards. There are Surgical, Gynecological, Medical, Pediatric, Ophthalmologic, Orthopedic and other departments in the hospital. All the procedures for isolation and antimicrobial susceptibility tests were performed at Amhara Regional Health Laboratory and Research Center. It is a well established and accredited laboratory center by Ethiopian drug administration and control authority.

\section{Study Design And Participants}

A cross-sectional study was conducted among randomly selected symptomatic UTI patients aged 5-15 years who presented to Felege Hiwot Referral Hospital. The sample size was determined using a single population proportion formula with the following assumption: proportion of symptomatic UTI in the population to be $21.5 \%{ }^{10}$ margin of sampling error tolerated 5\% (0.05), and critical value at $95 \%$ confidence interval of certainty (1.96), and $10 \%$ for non-response making the final sample size of 259 children.

Participants were selected consecutively among children's visiting pediatric departments until required samples were obtained.

The study was cleared from the ethical review board of Addis Ababa University. Official recognition and support was secured from the relevant organizations and departments. The interviewees have also been informed about the aim of the study. Additionally, written and verbal consent were secured from the study participants and/or caregivers. Finally, confidentiality was ascertained by justifying that no information was disclosed individually without the full willingness of the respondent.

\section{Data Collection Procedure} Clinical Examination

After obtaining an informed consent from children's parent, socio-demographic data and clinical data were collected from patient chart using pre-structured questioners by nurses.

\section{Specimen Collection}

A freshly voided midstream urine sample $(10-20 \mathrm{~mL})$ was collected in a wide mouthed sterile container after instructing the parents or guardians of enrolled children to clean their genitals with soap and water. 


\section{Culturing And Identification Procedure}

Urine specimens obtained from children were inoculated on blood, MacConkey and CLED agar (Oxoid, Ltd., Basingstoke, Hampshire, England) by using a calibrated loop $(0.001 \mathrm{~mL})$. Cultures were incubated in aerobic atmosphere at $37^{\circ} \mathrm{C}$ for $24 \mathrm{hrs}$. A positive urine culture was defined as colony count $\geq 10^{5} \mathrm{CFU} / \mathrm{mL}$ for midstream urine. A Stuart scientific colony counter was used for counting. All positive cultures were further identified by their colony characteristics, Gram staining was done to identify Gram positives from Gram negatives. Final confirmation was done by their pattern of biochemical reactions and rapid identification methods using the standard microbiological procedures.

\section{Antimicrobial Susceptibility Testing}

The antimicrobial susceptibility testing of all isolates was done by the standard Kirby-Bauer disk diffusion method, ${ }^{11}$ using commercial disks (Oxoid) according to Clinical and Laboratory Standards Institute (CLSI). Briefly, when pure culture was obtained, a loopful of bacteria was taken from a colony and transferred to a tube containing $5 \mathrm{~mL}$ of normal saline and mixed gently until it forms a homogenous suspension. Then, the turbidity of the suspension was adjusted to the density of a McFarland 0.5 (Mary-1'Etoil, France) in order to standardize the inoculum size. A sterile cotton swab was dipped into the suspension and the excess was removed by gentle rotation of the swab against the surface of the tube. The swab was then used to distribute the bacteria evenly over the entire surface of MuellerHinton agar (Oxoid). The inoculated plates were left at room temperature to dry for 3-5 mins. With the aid of sterile forceps, the following concentration of antibiotic discs were put on the surface of Mueller-Hinton agar (Oxoid): ampicillin $(10 \mu \mathrm{g})$, amoxicillin clavulanic acid $(30 \mu \mathrm{g})$, ciprofloxacin $(5 \mu \mathrm{g})$, gentamicin $(10 \mu \mathrm{g})$, ceftriaxone $(30 \mu \mathrm{g})$, nalidixic acid $(30 \mu \mathrm{g})$, nitrofurantoin $(300 \mu \mathrm{g})$, cefoxitin $(1 \mu \mathrm{g})$, trimethoprim-sulfamethoxazole $(25 \mu \mathrm{g})$ and ceftazidime $(30 \mu \mathrm{g})$. These antimicrobial agents were selected because of the frequent empirical treatment used by the clinicians. The plates were incubated at $37^{\circ} \mathrm{C}$ for 24 hrs. The result was interpreted as resistant, intermediate and sensitive based on CLSI guidelines. Multidrug resistance was defined as resistance to two or more of the antimicrobials tested. Positive results from urine culture and antimicrobial sensitivity test results were reported to the attending physician for subsequent treatment and follow up.

\section{Data Processing And Analysis}

Quantitative data were cleaned, edited and entered onto Epi-data version 3.2.1 and exported to the Statistical Package for the Social Sciences (SPSS) version 20 statistical software for further analysis. Fisher's exact test and binary logistic regression test results were used. A $P$-value less or equal to 0.05 was employed to declare the statistical significance.

\section{Results}

\section{Socio-Demographic Characteristics}

Of the 259 children investigated, 83 (32\%) were males and $176(68 \%)$ were females. We observed a mean age of 12 with $179(69.1 \%)$ of the study subjects between 11 and 15 years and the remaining were between 5 and 10 years. Majority of the study subjects were from rural areas 161 $(62.2 \%)$ (Table 1$)$.

\section{Culture Results}

Significant bacteriuria was observed in 41 of 259 (15.8\%) urine samples cultured. Of these, 11 (26.8\%) were Grampositive and $30(73.2 \%)$ were Gram-negative bacteria. Out of total positives, $9(22 \%)$ were from males and $32(78 \%)$ were from females $(\mathrm{P}=0.18)$. Twenty-eight $(68.3 \%)$ of children with significant bacteriuria were between 11 and 15 years while $13(31.7 \%)$ of them were between 5 and 10 years $(\mathrm{P}=0.9)$. Nine $(21.9 \%)$ of children with significant bacteriuria have parents with previous history of UTI and the rest $32(79.1 \%)$ did not have a parent with a history of UTI. Interestingly, there was statistical significant association between the rate of UTI and previous history of UTI in children's parents $(\mathrm{p}<0.05)$. (Table 2$)$.

Table I Sociodemographic Characteristics Of The Study Subjects Investigated For UTI In Felege Hiwot Referral Hospital (February 20I3 To May 20I3)

\begin{tabular}{|l|l|l|}
\hline Variables & Frequency & Percentage \\
\hline Sex & & \\
Male & 83 & 32 \\
Female & 176 & 68 \\
\hline Age & & \\
$5-10$ & 80 & 30.9 \\
II-15 & 179 & 69.1 \\
\hline Residence & & \\
Urban & 98 & 37.8 \\
Rural & 161 & 62.2 \\
\hline
\end{tabular}


Table 2 The Isolation Rate Of UTI In Children Attending Felege Hiwot Referral Hospital In Relation With Their Associated Risk Factors And Gram Stain (February 2013 To May 2013)

\begin{tabular}{|c|c|c|c|c|}
\hline \multirow[t]{3}{*}{ Category } & \multicolumn{4}{|c|}{ Significant Bacteriuria } \\
\hline & Yes & No & & \\
\hline & $\mathbf{N}(\%)$ & $\mathbf{N}(\%)$ & Total & P-value \\
\hline \multicolumn{5}{|l|}{ Sex } \\
\hline Male & $9(22)$ & $74(33.9)$ & 83 & 0.18 \\
\hline Female & $32(78)$ & $144(66.1)$ & 176 & \\
\hline \multicolumn{5}{|l|}{ Age (years) } \\
\hline $5-10$ & $13(3 \mid .7)$ & $67(30.7)$ & 80 & 0.9 \\
\hline $11-15$ & $28(68.3)$ & $151(69.3)$ & 179 & \\
\hline \multicolumn{5}{|c|}{ Urinary tract abnormality in children } \\
\hline Yes & $6(14.6)$ & $4 \mid(18.8)$ & 47 & 0.6 \\
\hline No & $35(85.4)$ & $177(8 \mid .2)$ & 212 & $35(85.4)$ \\
\hline \multicolumn{5}{|c|}{ History of UTI in parents } \\
\hline Yes & $9(21.9)$ & $23(10.6)$ & 32 & 0.034 \\
\hline No & $32(79.1)$ & $195(89.4)$ & 227 & \\
\hline \multicolumn{5}{|c|}{ Previous history of catheterization } \\
\hline Catheterized & $0(0)$ & $3(1.4)$ & 3 & 0.6 \\
\hline Non-catheterized & $4 \mathrm{I}(100)$ & $215(98.6)$ & 256 & \\
\hline \multicolumn{5}{|l|}{ Gram stain } \\
\hline Gram positive bacteria & II (26.8) & - & 11 & \\
\hline Gram negative bacteria & $30(73.2)$ & - & 30 & \\
\hline Total & $41(100)$ & $218(100)$ & $259(100)$ & \\
\hline
\end{tabular}

\section{Bacterial Etiologies}

A total of 41 uropathogens were isolated. Out of this, 11 (26.8\%) were Gram-positive bacteria and 30 (73.2\%) were Gram-negative bacteria. E. coli 14 (34.1\%) was the most frequently isolated uropathogen followed by Pseudomonas spp. $9(22.0 \%)$ and S. saprophyticus $6(14.6 \%)$. Among Gram-positive bacteria, S. saprophyticus $6(54.6 \%)$ was the most frequently isolated bacteria followed by $S$. aureus $3(27.3 \%)$ as shown in Figure 1.

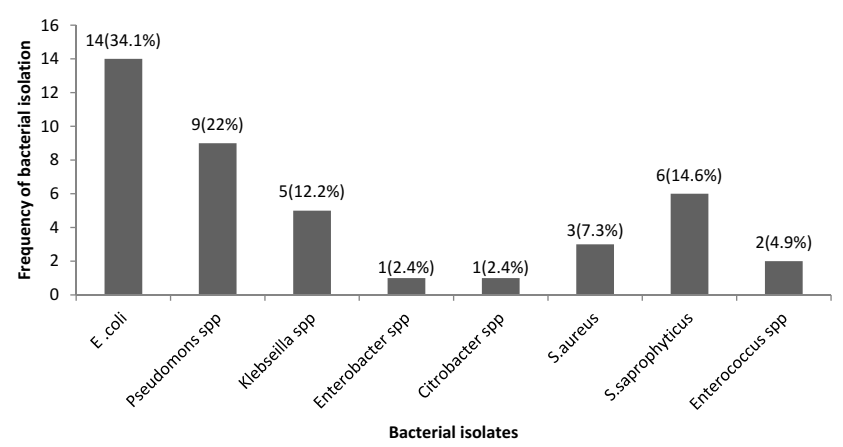

Figure I Distribution of uropathogens isolated from children attending Bahir Dar Felege Hiwot Referral Hospital, February 2013 to May 2013.

\section{Antimicrobial Susceptibility Testing}

Antimicrobial susceptibility test was performed for all urine culture positive samples. Gram-negative bacteria isolated were tested against 10 antibiotics while Grampositive isolates were tested against 7 antibiotics via disk diffusion (Tables 3 and 4, respectively).

Overall, the percentage of sensitivity for Gram-negative were high for ciprofloxacin (70\%), norfloxacin (63.4\%) and for ceftriaxone $(60 \%)$ among selected antibiotics, whereas a high level of resistance was observed for ampicillin (80\%) and nitrofurantoin (70\%). E. coli, the most frequently isolated bacteria, was highly resistant for ampicillin (100\%). Furthermore, more than $80 \%$ of Gram-negative isolates showed multiple resistances for antibiotics (Table 3).

Overall, the percentage of sensitivity for Gram-positive bacterial isolates were relatively high for ciprofloxacin, penicillin and erythromycin, each accounted 7/9 (77.8\%), 8/11 (72.7\%) and 8/11 (72.7\%), respectively. Overall resistance was high for ampicillin 9/11 (81.8\%). All isolates showed multiple resistances to the antibiotics tested (100\%) (Table 4). 


\begin{tabular}{|c|c|c|c|c|c|c|}
\hline$\tilde{y}$ & 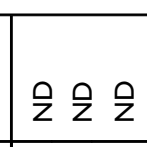 & 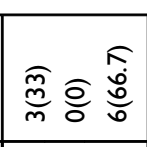 & $2 \% \frac{1}{2}$ & 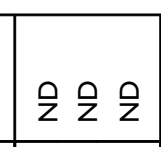 & $\frac{2}{2} \frac{2}{2}$ & 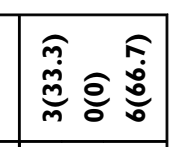 \\
\hline$\frac{\pi}{2}$ & 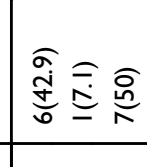 & $\frac{\rho}{2} \frac{\rho}{2}$ & 畜亯宫 & ㅎㅎㅇㅎㅎㅇ흥 & 홍ㅎㅎㅇ흥 & \\
\hline \begin{tabular}{|l}
$\frac{\alpha}{2}$ \\
$\frac{0}{2}$ \\
\end{tabular} & 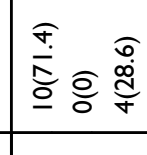 & 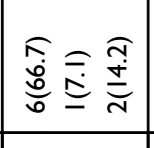 & 亭豆哥 & 呞豆 & 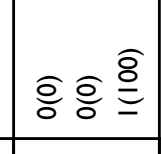 & \\
\hline 离 & 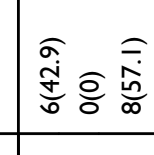 & 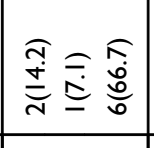 & 高宫高 & 离合合 & 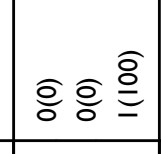 & 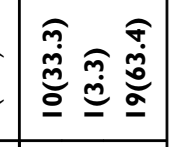 \\
\hline 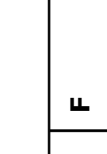 & 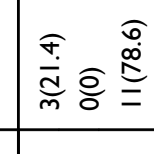 & 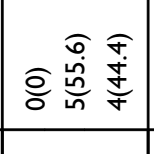 & 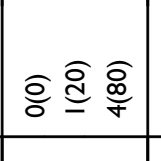 & 홍ㅎㅎㅇ흥 & 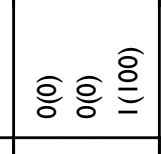 & $\frac{\mathrm{g}}{\mathrm{m}}$ \\
\hline$z$ & 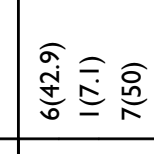 & 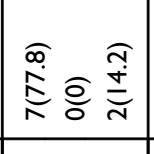 & 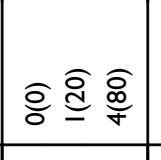 & 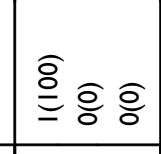 & 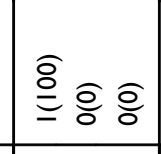 & 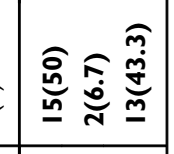 \\
\hline$\frac{0}{\frac{2}{4}}$ & 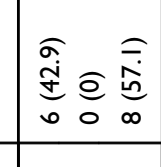 & 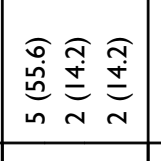 & 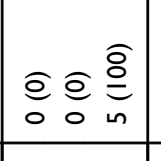 & 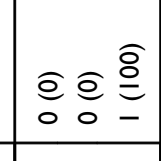 & 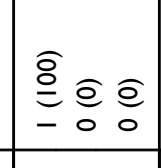 & \\
\hline$\stackrel{8}{8}$ & 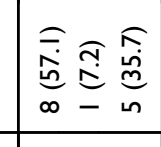 & 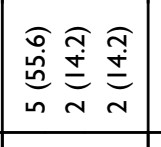 & 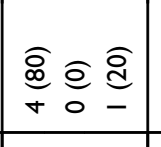 & 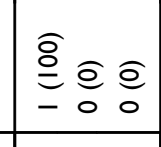 & 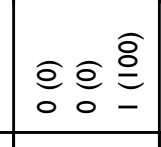 & 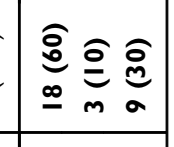 \\
\hline 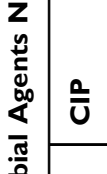 & 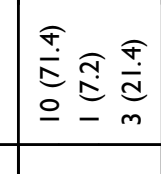 & 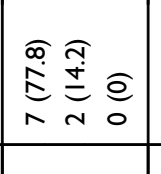 & 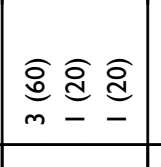 & 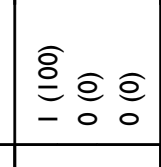 & 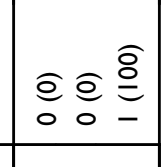 & \\
\hline 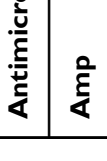 & $\mid \begin{array}{l}0 \\
0 \\
0\end{array}$ & 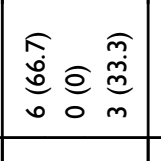 & 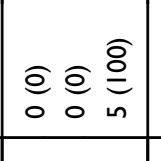 & 就言高 & 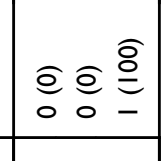 & 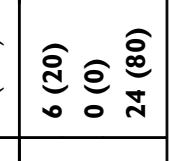 \\
\hline & $n-\alpha$ & $n-\infty$ & $n-\infty$ & $n-\infty$ & $n-\alpha$ & $n-\infty$ \\
\hline 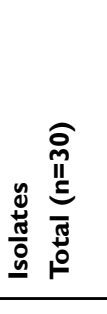 & & 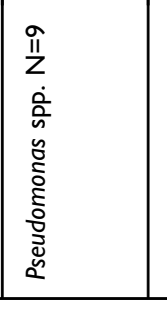 & 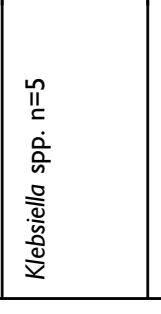 & 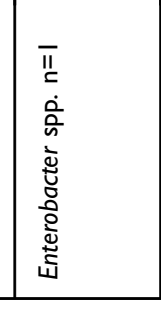 & & 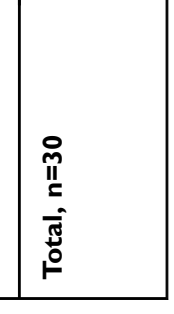 \\
\hline
\end{tabular}


Table 4 Antimicrobial Susceptibility Pattern Of Gram-Positive Bacteria Isolated From Children With UTI Attending Felege Hiwot Referral Hospital (February 2013 To May 2013)

\begin{tabular}{|c|c|c|c|c|c|c|c|c|}
\hline \multirow[t]{2}{*}{ Isolates } & \multicolumn{8}{|c|}{ Antimicrobial Agents N (\%) } \\
\hline & & AMP & Ox & $\mathbf{F}$ & $\mathbf{P}$ & SXT & CIP & $\mathbf{E}$ \\
\hline S. aureus $\mathrm{N}=3$ & $\begin{array}{l}S \\
\mathrm{I} \\
\mathrm{R}\end{array}$ & $\begin{array}{l}0(0) \\
0(0) \\
3(100)\end{array}$ & $\begin{array}{l}2(66.7) \\
0(0) \\
I(33.3)\end{array}$ & $\begin{array}{l}2(66.7) \\
0(0) \\
I(33.3)\end{array}$ & $\begin{array}{l}0(0) \\
0(0) \\
3(100)\end{array}$ & $\begin{array}{l}0(0) \\
0(0) \\
3(100)\end{array}$ & $\begin{array}{l}3(100) \\
0(0) \\
0(0)\end{array}$ & $\begin{array}{l}2(66.7) \\
0(0) \\
(133.3)\end{array}$ \\
\hline S. saprophyticus $\mathrm{N}=6$ & $\begin{array}{l}S \\
\mathrm{I} \\
\mathrm{R}\end{array}$ & $\begin{array}{l}\text { I (16.7) } \\
0(0) \\
5(83.3)\end{array}$ & $\begin{array}{l}4(66.7) \\
0(0) \\
2(33.3)\end{array}$ & $\begin{array}{l}2(33.3) \\
2(33.3) \\
2(33.3)\end{array}$ & $\begin{array}{l}6(100) \\
0(0) \\
0(0)\end{array}$ & $\begin{array}{l}6(100) \\
0(0) \\
0(0)\end{array}$ & $\begin{array}{l}4(66.7) \\
0(0) \\
2(33.3)\end{array}$ & $\begin{array}{l}5(83.3) \\
0(0) \\
I(16.7)\end{array}$ \\
\hline Enterococcus spp. $\mathrm{N}=2$ & $\begin{array}{l}S \\
\mathrm{I} \\
\mathrm{R}\end{array}$ & $\begin{array}{l}\text { I (50) } \\
0(0) \\
\text { I (50) }\end{array}$ & $\begin{array}{l}\text { ND } \\
\text { ND } \\
\text { ND }\end{array}$ & $\begin{array}{l}0(0) \\
2(100) \\
0(0)\end{array}$ & $\begin{array}{l}2(100) \\
0(0) \\
0(0)\end{array}$ & $\begin{array}{l}2(100) \\
0(0) \\
0(0)\end{array}$ & $\begin{array}{l}\text { ND } \\
\text { ND } \\
N D\end{array}$ & $\begin{array}{l}I(50) \\
0(0) \\
I(50)\end{array}$ \\
\hline Total, $\mathbf{N}=$ I I & $\begin{array}{l}\mathbf{S} \\
\mathbf{I} \\
\mathbf{R}\end{array}$ & $\begin{array}{l}2(18.2) \\
0(0) \\
9(8 \mid .8)\end{array}$ & $\begin{array}{l}6(66.7) \\
0(0) \\
3(33.3)\end{array}$ & $\begin{array}{l}4(36.4) \\
4(36.4) \\
3(27.2)\end{array}$ & $\begin{array}{l}8(72.8) \\
0(0) \\
3(27.2)\end{array}$ & $\begin{array}{l}8(72.8) \\
0(0) \\
3(27.2)\end{array}$ & $\begin{array}{l}7(77.8) \\
0(0) \\
2(22.2)\end{array}$ & $\begin{array}{l}8(72.7) \\
0(0) \\
3(27.2)\end{array}$ \\
\hline
\end{tabular}

Abbreviations: AMP, ampicillin; ox, cefoxitin; F, nitrofurantoin; p, penicillin; SXT, trimethoprim sulfamethoxazole; CIP, ciprofloxacin; E, erythromycin; CL, clindamycin; ND, not done.

Multidrug resistance (MDR) to two or more drugs was observed for 25/30 (83.3\%) of Gram-negative bacteria and 11/ 11 (100\%) Gram-positive bacteria. Table 5 shows the overall prevalence of MDR in both groups was $37 / 41$ (90.3\%).

\section{Discussion}

In the present study, the overall prevalence of UTI in children was $15.8 \%$. It is comparable with the study

Table 5 Multidrug Resistance Pattern Of Gram-Positive And Gram-Negative Bacteria Isolated From Urine Culture Of Children Attending Felege Hiwot Referral Hospital (February 2013 To May 2013)

\begin{tabular}{|l|l|}
\hline $\begin{array}{l}\text { Number Of Isolated Gram-Negative } \\
\text { Bacteria }\end{array}$ & $\begin{array}{l}\text { Number Of MDR, } \mathbf{n} \\
\mathbf{( \% )}\end{array}$ \\
\hline E. coli $(\mathrm{n}=14)$ & $13(92.9)$ \\
Pseudomonas spp. $(\mathrm{n}=9)$ & $5(55.6)$ \\
Klebsiella spp. $(\mathrm{n}=5)$ & $5(100)$ \\
Enterobacter spp. $(\mathrm{n}=\mathrm{I})$ & $\mathrm{I}(100)$ \\
Citrobacter spp. $(\mathrm{n}=\mathrm{I})$ & $\mathrm{I}(100)$ \\
Sub-total $(\mathrm{n}=30)$ & $25(83.3)$ \\
\hline Number Of Isolated Gram-Positive & Number Of MDR, $\mathbf{n}$ \\
Bacteria & $\mathbf{( \% )}$ \\
\hline S. aureus $(\mathrm{n}=3)$ & $3(100)$ \\
S. saprophyticus $(\mathrm{n}=6)$ & $6(100)$ \\
Enterococcus spp. $(\mathrm{n}=2)$ & $2(100)$ \\
Sub-total $(\mathrm{n}=1 \mathrm{I})$ & $11(100)$ \\
\hline Total $(\mathrm{n}=\mathbf{4 I})$ & $\mathbf{3 7}(\mathbf{9 0 . 3 )}$ \\
\hline
\end{tabular}

conducted in Tanzania, Nigeria and Gondar, 20.3\% ${ }^{12}$ $20.5 \%{ }^{13}$ and $17.5 \%,{ }^{14}$ respectively. A lower prevalence was reported from Turkey, $7.1 \%{ }^{4}$ India, $10.9 \%{ }^{15}$ Iran, $7.8 \%{ }^{16}$ Bahir Dar, $9.4 \%{ }^{17}$ and Addis Ababa, $11.6 \%{ }^{18}$ The variation might be due to difference in sample size and habit of personal hygiene.

Whereas a higher prevalence was reported in Italy, $22.6 \%{ }^{19}$ People's Republic of China, 36.5\% ${ }^{20}$ Nepal, $45.5 \%^{21}$ Gondar, $28.1 \%{ }^{22}$ and another study in Cameron also showed $58.3 \%{ }^{23}$ This difference might be due to difference in age of the study subjects, sample size and presence of complication, for example, in the study conducted in People's Republic of China with nephritic syndrome.

In the present study, Gram-negative bacteria were predominantly isolated with a rate of $73.2 \%$ which is in agreement with Madagascar, $65.5 \%{ }^{24}$ Kenya, $78.9 \%{ }^{25}$ and Bahir Dar, $61.9 \%,{ }^{17}$ but lower than in a study reported from Mekelle, $83 \%{ }^{26}$ India, $92 \%{ }^{15}$ and South Africa which is $87.5 \% .{ }^{27}$ In most studies conducted elsewhere in the world, the commonest uropathogens were Gramnegative bacteria. The frequency of isolation of uropathogens varies with different geographical locations.

Among the overall bacterial isolates, E. coli $34.1 \%$ was the most frequently isolated uropathogens in our study. Our study agrees with other studies conducted in Iran, ${ }^{28}$ India, ${ }^{15}$ Germany, ${ }^{29}$ Tanzania, $^{12}$ Gondar $^{22}$ and TikurAnbesa Specialized Hospital, ${ }^{18}$ but in a study conducted in People's 
Republic of China, ${ }^{20}$ Nairobi $^{30}$ and Jimma, ${ }^{8}$ Enterococcus spp., S. aureus and Klebsiella spp. were the dominant pathogens isolated, respectively. Generally in the present study and in most other studies conducted elsewhere, E. coli was the most frequently isolated uropathogens. It is the most common cause of UTI and accounts for $75-90 \%$ of UTIs in children. However, its relative frequency varies in different areas. ${ }^{31} E$. coli is the commonest flora of the gastrointestinal tract and bowel from which it ascends to urinary tract and it has well characterized virulence factors to colonize the urinary tracts. The second leading bacterial isolate in our study was Pseudomonas spp. (22\%) followed by S. saprophyticus (14.6\%). But in other studies reported Klebsiella spp., $S$. aureus and Enterococcus faecalis were the second leading uropathogens in Iran, ${ }^{28}$ Germany ${ }^{29}$ and TikurAnbesa specialized hospital, ${ }^{8}$ respectively.

The first leading bacterium among Gram positives was S. Saprophyticus (54.6\%) followed by S. aureus (27.3\%). Our study disagrees with Germany ${ }^{29}$ Enterococcus spp.; at Gondar $^{32}$ and Bahir Dar, ${ }^{17}$ S. aureus were the first leading pathogens. Bacterial etiologies vary with different geographical regions even vary over time within a population.

In the present study from children having significant bacteriuria, $21.9 \%$ of them have parents with previous history of UTI $(\mathrm{p}<0.05)$. Similarly in a study conducted in Turkey showed that children with a family history of UTI had a 3.8-fold risk of developing UTI $(\mathrm{P}<0.05)$ compared to those without. ${ }^{4}$ Family history of UTI is the risk factor for UTI in children. Because some abnormalities and renal defects in parents may be acquired by children congenitally. Example children acquired vulvo - urethral reflux before birth.

In the present study, sensitivity of Gram-negative bacteria were relatively high for ciprofloxacin (70\%), norfloxacin $(63.4 \%)$ and ceftriaxone $(60 \%)$. A study in $\mathrm{Jimma}^{8}$ is in agreement with our study which showed that ciprofloxacin controlled $75 \%$ of Gram-negative bacteria and in Gondar $^{14}$ ciprofloxacin was relatively best drug. In other study, ciprofloxacin is a widely used fluoroquinolone with high bacterial activity against uropathogens and well-established clinical efficacy in the treatment of UTIs. ${ }^{33}$

High level of resistance of Gram-negative bacteria was also observed for ampicillin (80\%) and nitrofurantoin $(70 \%)$ in the present study. This is in agreement with the study in India ${ }^{15}$ which showed high level of resistance to ampicillin; Mekelle $(>80 \%)^{26}$ and $\mathrm{Jimma}^{8}(88 \%)$ of the isolates were resistant to ampicillin. In contrast with our study, Gram-negative isolates were susceptible to nitrofurantoin in studies conducted in Jimma $75 \%{ }^{26}$ and Addis Ababa $>85 \% .{ }^{18}$ Availability and use of these drugs in the community may vary in different areas.

E. coli, the first frequently isolated bacterium, was $(100 \%)$ resistant for ampicillin and $(78.6 \%)$ for nitrofurantoin. Our study is comparable with India ${ }^{15}$ that resistance for nitrofurantoin was $(80 \%)$. On the contrary in a study conducted in Iran, ${ }^{7}$ nitrofurantoin had the highest activity against E.col. On the other hand, ampicillin resistance against $E$. coli in Tanzania ${ }^{12}$ revealed similar finding with the present study. The high resistance of Gram-negative bacteria to ampicillin ' might be due to ease availability, frequently prescribed drug and its low cost.

In the present study the overall percentage of sensitivity for Gram-positive isolates were high for penicillin, ciprofloxacin and erythromycin, 72.8\%, 77.8\% and $72.8 \%$, respectively. Our study is almost comparable with Gondar $^{14}$ and Addis Ababa. ${ }^{18}$ Whereas (>80\%) Grampositive isolates were resistant for ampicillin and cotrimoxazole. The present study disagrees with that of Gondar where ampicillin and cotrimoxazole resistance were $17.1 \%$ and $57.1 \%$, respectively. ${ }^{14}$

All isolated $S$. aureus were resistant for ampicillin, penicillin and cotrimoxazole. A study in Gondar showed that among Gram positives, S.aureus showed high level of resistance for cotrimoxazole (53.3\%), ampicillin (43\%) and penicillin $(36.7 \%){ }^{22}$ The present study is almost comparable with this study.

Multidrug resistance (MDR) to two or more drugs was observed in $83.3 \%$ and $100 \%$ of Gram-negative and Grampositive isolates, respectively. Overall, MDR in both groups was $90.3 \%$. This study is almost similar with the study in Bahir Dar which was $93.1 \%{ }^{34}$ and in Gondar, $85.7 \%{ }^{22}$ This high MDR in the present study might be due to widespread misuse of antibiotics, inappropriate prescription of drugs and lack of knowledge about drug resistance in the study area.

\section{Limitation Of The Study}

Our study has some limitations like antimicrobial susceptibility test was not done because of getting antibiotics disks and few bacterial isolates were not identified at a species level. The other limitation was using only Kirby-Bauer method to perform antimicrobial susceptibility instead of micro-dilution method, which makes more sensitive.

\section{Conclusion}

The result showed that the most predominant bacterial isolate was E. coli. Overall, the percentage of sensitivity of Gram- 
negative bacteria to all antibiotics tested was relatively low, except for ciprofloxacin, norfloxacin and ceftriaxone, which were relatively high; whereas Gram-positive bacteria showed relatively high percentage of sensitivity for ciprofloxacin, penicillin and erythromycin among selected antibiotics tested in the study. Therefore, these antibiotics may be used for empirical therapy of urinary tract infection (UTI) when culture and sensitivity are unavailable.

The present study also showed a high incidence of resistance to most of the antimicrobial agents tested. The prevalence of multiple drug resistance was also high. This calls for a continuous monitoring and review of antimicrobial policy in the hospital and the country at large.

\section{Abbreviations}

AAU, Addis Ababa University; FHRH, Felege Hiwot Referral Hospital; ARHRLC, Amhara Regional Health Research Laboratory Center; CAUTIs, catheter-associated urinary tract infection; CFU, colony-forming unit; CLED, Cysteine Lactose Electrolyte Deficient media; CI, confidence interval; CLSI, Clinical \& Laboratory Standards Institute; ESBL, extended-spectrum beta-lactamases; MDR, multiple drug resistance; OPD, outpatient department; UTI, urinary tract infection; TMP-SMX, trimethoprim sulfamethoxazole.

\section{Ethics Approval And Consent To Participate}

The MSc research project proposal was ethically cleared by the Department Research and Ethical Review Committee and was approved by the Department of Microbiology, Immunology and Parasitology, School of Medicine, Addis Ababa University. Official permission from the study site was obtained. Written informed consent was obtained from each child's parents/guardians. Urine culture and antimicrobial sensitivity test results were reported to the attending physician for subsequent treatment and follow up.

\section{Availability Of Data And Materials}

The data that support the findings of this study will be available from the corresponding author upon reasonable request in the form of Statistical Package for the Social Sciences (SPSS).

\section{Acknowledgments}

The authors would like to thank Addis Ababa University, Department of Microbiology, Immunology and Parasitology,
Faculty of Medicine for sponsoring the research and Amhara Regional Health Laboratory Research Center for unreserved material and reagent supply that made the study possible. We extend our gratitude to physicians in the Department of Pediatrics OPD at Felege Hiwot Referral Hospital (FHRH), FHRH medical director, and all laboratory staff, for their support in screening of symptomatic study subjects and organizing the preconditions of sample collection and data collection. We also extend profound gratitude to the study subjects and their parents, without whom this research work would not have been possible.

\section{Authors Contributions}

YB: Principal investigator of the study, study design, data collection, laboratory work, data analysis and drafted the manuscript; DA and YWA: study design, supervision of data collection, data analysis, and drafted the manuscript; GY and AG: critically reviewed the data and drafted the manuscript. All authors gave final approval of the version to be published, and agree to be accountable for all aspects of the work.

\section{Funding}

This study was sponsored by Addis Ababa University, Addis Ababa, Ethiopia.

\section{Disclosure}

The authors declare that they have no conflicts of interest in this work.

\section{References}

1. Weichhart T, Haidinger M, Hörl WH, Säemann MD. Current concepts of molecular defence mechanisms operative during urinary tract infection. Eur J Clin Invest. 2008;38(s2):29-38. doi:10.1111/j.1365-2362.2008.02006.x

2. Olson RP, Harrell LJ, Kaye KS. Antibiotic resistance in urinary isolates of escherichia coli from college women with urinary tract infections. Antimicrob Agents Chemother. 2009;53(3):1285-1286. doi:10. 1128/AAC.01188-08

3. Freedman AL. Urologic diseases in north america project: trends in resource utilization for urinary tract infections in children. $J$ Urol. 2005;173(3):949-954. doi:10.1097/01.ju.0000152092.03931.9a

4. Zincir H, Erten ZK, Özkan F, Sevi ̌̆ Ü, Başer M, Elmalı F. Prevalence of urinary tract infections and its risk factors in elementary school students. Urol Int. 2012;88(2):194-197. doi:10.1159/000335554

5. Manges AR, Natarajan P, Solberg OD, Dietrich PS, Riley LW. The changing prevalence of drug-resistant Escherichia coli clonal groups in a community: evidence for community outbreaks of urinary tract infections. Epidemiol Infect. 2006;134(2):425-431. doi:10.1017/ S0950268805005005

6. Kahan NR, Chinitz DP, Waitman D-A, Dushnitzky D, Kahan E, Shapiro M. Empiric treatment of uncomplicated urinary tract infection with fluoroquinolones in older women in israel: another lost treatment option? Ann Pharmacother. 2006;40(12):2223-2227. doi:10.1345/aph.1H396 
7. Mortazavi F, Shahin N. Changing patterns in sensitivity of bacterial uropathogens to antibiotics in children. Pak J Med Sci. 2009;25(5):801805.

8. Teshager L, Asrat D, Gebre-Selassie S, Tamiru S. Catheterized and non-catheterized urinary tract infections among patients attended at Jimma University Teaching Hospital, Southwest, Ethiopia. Ethiop Med J. 2008;46(1):55-62.

9. Emiru T, Beyene G, Tsegaye W, Melaku S. Associated risk factors of urinary tract infection among pregnant women at Felege Hiwot Referral Hospital, Bahir Dar, North West Ethiopia. BMC Res Notes. 2013;6(1):292. doi:10.1186/1756-0500-6-292

10. Arya SC, Agarwal N. Urinary tract infection in febrile children with sickle cell anaemia. Ann Trop Paediatr. 2003;23(3):221-229. doi:10.1179/027249303322296556

11. Bauer AW, Kirby WM, Sherris JC, Turck M. Antibiotic susceptibility testing by a standardized single disk method. Am J Clin Pathol. 1966;45(4):493-496. doi:10.1093/ajcp/45.4_ts.493

12. Msaki BP, Mshana SE, Hokororo A, Mazigo HD, Morona D. Prevalence and predictors of urinary tract infection and severe malaria among febrile children attending Makongoro health centre in Mwanza city, North-Western Tanzania. Arch Public Health. 2012;70(1):4. doi:10.1186/0778-7367-70-4

13. Mava Y, Timothy SY, Zailani SB, Ambe JP. Significance of Pyuria in the diagnosis of urinary tract infection in children with sickle cell anaemia in Maiduguri, Nigeria. Afr J Clin Exp Microbiol. 2012;13 (2):103-109. doi:10.4314/ajcem.v13i2.8

14. Yismaw G, Asrat D, Woldeamanuel Y, Unakal CG. Urinary tract infection: bacterial etiologies, drug resistance profile and associated risk factors in diabetic patients attending Gondar University Hospital, Gondar, Ethiopia. Eur J Exp Biol. 2012;2(4):889-898.

15. Akram M, Shahid M, Khan AU. Etiology and antibiotic resistance patterns of community-acquired urinary tract infections in $\mathrm{J} \mathrm{N} \mathrm{M} \mathrm{C}$ Hospital Aligarh, India. Ann Clin Microbiol Antimicrob. 2007;6:4. doi:10.1186/1476-0711-6-4

16. Mirsoleymani SR, Salimi M, Shareghi Brojeni M, Ranjbar M, Mehtarpoor M. Bacterial pathogens and antimicrobial resistance patterns in pediatric urinary tract infections: a four-year surveillance study (2009-2012). Int J Pediatr. doi:10.1155/2014/126142

17. Melaku S, Kibret M, Abera B, Gebre-Sellassie S. Antibiogram of nosocomial urinary tract infections in Felege Hiwot referral hospital, Ethiopia. Afr Health Sci. 2012;12(2):134-139. doi:10.4314/ahs.v12i2.9

18. Assefa A, Asrat D, Woldeamanuel Y, Abdella A, Melesse T. Bacterial profile and drug susceptibility pattern of urinary tract infection in pregnant women at Tikur Anbessa Specialized Hospital Addis Ababa, Ethiopia. Ethiop Med J. 2008;46(3):227-235.

19. Magliano E, Grazioli V, Deflorio L, et al. Gender and age-dependent etiology of community-acquired urinary tract infections. Sci World J. doi: $10.1100 / 2012 / 349597$

20. Song SN, Zhang BL, Wang WH, Zhang X. [Spectrum and drug sensitivity of pathogenic bacteria in children with nephrotic syndrome complicated by urinary tract infection: an analysis of 97 cases]. Zhongguo Dang Dai Er Ke Za Zhi Chin J Contemp Pediatr. 2012;14(9):657-660.
21. Singh SD, Madhup SK. Clinical profile and antibiotics sensitivity in childhood urinary tract infection at Dhulikhel Hospital. Kathmandu Univ Med J. 2013;11(4):319-324. doi:10.3126/kumj.v11i4.12541

22. Tessema B, Kassu A, Mulu A, Yismaw G. Pridominant isolates of urinary tract pathogens and their antimicrobial susceptibility patterns in Gondar University Teaching Hospital, northwest Ethiopia. Ethiop Med J. 2007;45(1):61-67.

23. Akoachere J-FTK, Yvonne S, Akum NH, Seraphine EN. Etiologic profile and antimicrobial susceptibility of community-acquired urinary tract infection in two Cameroonian towns. BMC Res Notes. 2012;5(1):219. doi:10.1186/1756-0500-5-219

24. Rasamiravaka T, Shaista Sheila HSL, Rakotomavojaona T, Rakoto-Alson AO, Rasamindrakotroka A. Changing profile and increasing antimicrobial resistance of uropathogenic bacteria in Madagascar. Médecine Mal Infect. 2015;45(5):173-176. doi:10.1016/j.medmal.2015.03.006

25. Okwara FN, Obimbo EM, Wafula EM, Murila FV. Bacteraemia, urinary tract infection and malaria in hospitalised febrile children in Nairobi: is there an association? East Afr Med J. 2004;81(1):47-51. doi:10.4314/eamj.v81i1.8795

26. Tesfahunegn Z, Asrat D, Woldeamanuel Y, Estifanos K. Bacteriology of surgical site and catheter related urinary tract infections among patients admitted in Mekelle Hospital, Mekelle, Tigray, Ethiopia. Ethiop Med J. 2009;47(2):117-127.

27. Jeena PM, Coovadia HM, Adhikari MA. Bacteriuria in children attending a primary health care clinic: a prospective study of catheter stream urine samples. Ann Trop Paediatr. 1996;16(4):293-298. doi:10.1080/02724936.1996.11747841

28. Navidinia M, Karimi A, Rahbar M, et al. Study prevalence of verotoxigenic E.coli isolated from Urinary Tract Infections (UTIs) in an Iranian Children Hospital. Open Microbiol J. 2012;6:1-4. doi:10.2174/1874285801206010001

29. Pape L, Gunzer F, Ziesing S, Pape A, Offner G, Ehrich JH. [Bacterial pathogens, resistance patterns and treatment options in community acquired pediatric urinary tract infection]. Klin Padiatr. 2004;216 (2):83-86. doi:10.1055/s-2004-823143

30. Adeleke SI, Asani MO. Urinary tract infection in children with nephrotic syndrome in Kano, Nigeria. Ann Afr Med. 2009;8(1):38. doi:10.4103/1596-3519.55762

31. Esbjörner E, Berg U, Hansson S. Association writing committee on behalf of the SPN. Epidemiology of chronic renal failure in children: a report from Sweden 1986 - 1994. Pediatr Nephrol. 1997;11 (4):438-442. doi:10.1007/s004670050312

32. Moges AF, Genetu A, Mengistu G. Antibiotic sensitivities of common bacterial pathogens in urinary tract infections at Gondar Hospital, Ethiopia. East Afr Med J. 2002;79(3):140-142. doi:10.4314/eamj. v79i3.8893

33. Blondeau JM. Current Issues in the Management of Urinary Tract Infections. Drugs. 2004;64(6):611-628. doi:10.2165/00003495-20046 4060-00004

34. Biadglegne F, Abera B. Antimicrobial resistance of bacterial isolates from urinary tract infections at Felge Hiwot Referral Hospital, Ethiopia. Ethiop J Health Dev. 2010;23:3. doi:10.4314/ejhd.v23i3.53248
Infection and Drug Resistance

\section{Publish your work in this journal}

Infection and Drug Resistance is an international, peer-reviewed openaccess journal that focuses on the optimal treatment of infection (bacterial, fungal and viral) and the development and institution of preventive strategies to minimize the development and spread of resistance. The journal is specifically concerned with the epidemiology of antibiotic resistance and the mechanisms of resistance development and diffusion in both hospitals and the community. The manuscript management system is completely online and includes a very quick and fair peerreview system, which is all easy to use. Visit http://www.dovepress.com/ testimonials.php to read real quotes from published authors. 